\title{
Better results with HIV clubs in South Africa
}

$\mathrm{W}$

hen nine people gathered one early morning in a tworoom plywood house in Khayelitsha, a shantytown suburb of Cape Town, the conversation mostly concerned a battered bathroom scale. Amid plenty of lighthearted banter, everyone took a turn to be weighed. In the corner of the room, a community health worker - part of a team from a nearby clinic operated by Médecins sans Frontières (MSF) - noted the results in a dog-eared National Health Laboratory Service ledger.

The occasion, explained Nontle Cwasi, a 47-year-old mother of two, was the bimonthly meeting of Adherence Club 66, one of hundreds of clubs in Khayelitsha that help patients with HIV manage their symptoms and pickup refills of antiretroviral drugs (ARVs) provided for free as part of the South African government's commitment to providing universal HIV treatment.

"By coming here, I can see friends, get my medicine, discuss my symptoms, and I can still get to work on time," Cwasi said. Before joining the club in 2013, she often queued for hours at a local clinic for drug refills and consultations. But the contrast between her experiences at the clinic and the adherence club is more than just a matter of efficiency, she said. At club meetings, there's a strong sense of solidarity, reassurance and openness.

"It's a relief to be able to talk calmly about having HIV in the same way people talk about having diabetes or high blood pressure. It's calming. It lifts the spirit. And it breaks the secrecy and shame that I used to feel living with HIV."

Dr. Lynne Wilkinson, the MSF field coordinator in Khayelitsha, explains that with an estimated $37 \%$ prevalence rate of HIV among the township's 400000 inhabitants, the adherence clubs aim to take some of the pressure off the medical system. "We now realize that treatment adherence and retention is the main challenge."

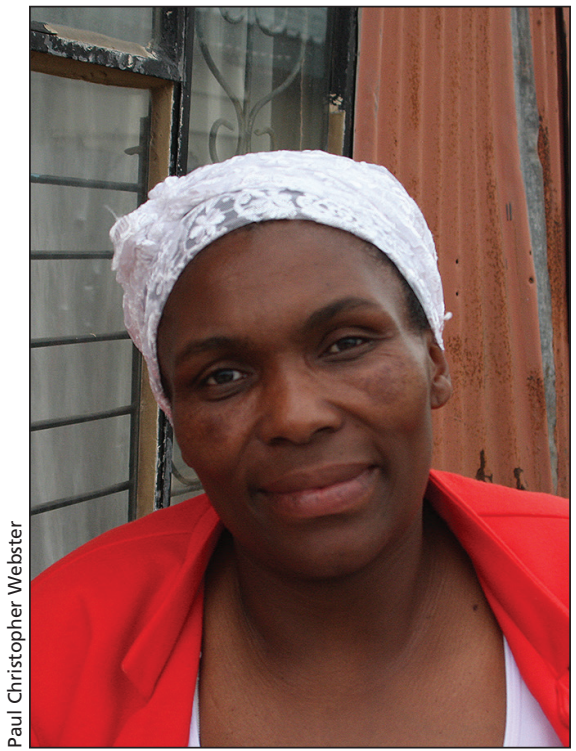

Nontle Cwasi says she attends twicemonthly adherence club meetings to help her manage her HIV and pick up free drugs.

Since being launched in 2011, the clubs now reach almost a third of all patients in Khayelitsha, which was the first community in Africa where largescale HIV treatment was implemented. Club membership is restricted to adults with stable weight who have been on the same ARV regime for at least 12 months with no detectable viral loads and no medical conditions requiring regular clinical consultations.

Last year, the club concept was adopted by the Cape Town Metro District Health Services to include 27800 people. As the national government watches closely, three more provinces are adopting the same care model.

With 6.2 million people infected about $18 \%$ of the population - South Africa has more people with HIV than any other country and hosts the world's largest ARV program. More than 2.5 million people are in treatment, but as the government moves the treatment threshold from a CD4 T-cell count of 350 per cubic millilitre to 500, that number is forecast to reach three million.
But at the same time, some foreign donors - most notably the US President's Emergency Plan for AIDS Relief - are phasing out support as South Africa reaches middle-income status.

Substantial cost savings are achieved by moving care out of public health clinics and in effect "demedicalizing" HIV treatment, Wilkinson said. In addition, clinicians are liberated to treat the highest-need patients, "at the same time as it creates a humanizing effect."

But the aim is not to reduce government accountability by moving toward volunteerism, she emphasizes. "The debate in discussions of demedicalization is where to draw the line. Because we still want the health system to be responsible for these patients. You get chaos otherwise: you get a breakdown in the referral system."

A Feb. 2013 study in PLoS One, suggests the adherence clubs are delivering better outcomes. Over a 40-month period, retention in the clubs in Khayelitsha was $97 \%$ versus $83 \%$ in clinics. Club participation also reduced virologic rebound in patients by $67 \%$.

At Groote Schuur Hospital in Cape Town, Chief Specialist Dr. Bongani Mayosi, agrees that adherence clubs represent "the model to follow in task shifting and task sharing to more community-based care." But to scale up the model, he warns, South Africa must trains hundreds of thousands of community health workers.

South Africa, with $0.7 \%$ of the world's population, accounts for $17 \%$ of the global burden of HIV infection, Mayosi notes. But with the national health budget increasingly skewed toward HIV treatment, the quality of health care as a whole is at risk. "We are going to overcome HIV/AIDS. But it will trigger more disasters if that means we overlook new epidemics of other diseases." - Paul Christopher Webster, Cape Town, South Africa

CMAJ 2015. DOI:10.1503/cmaj.109-5040 